\title{
Devnagari Phonetic Speech Analysis
}

\author{
D.S.Shete ${ }^{1}$, S.B. Patil ${ }^{2}$ \\ ${ }^{1}$ (Department Of Electronics Engineering, J.J.Magdum College of Engg. ,Jaysingpur, Shivaji University \\ Kolhapur,India) \\ ${ }^{2}$ (Department Of Electronics Engineering , D.Y.Patil College of Engg. \& Tech., Kolhapur, Shivaji University \\ Kolhapur,India)
}

\begin{abstract}
This paper presents a description of the work done on phonetic speech analysis. The work aims in generating phonetic codes of the uttered speech in training-less, human independent manner. This work is guided by the working of ear in response to audio signals. The Devnagri script inspires the work presented. The Devnagari script classifies and arranges 46 phonemes in a scientific manner based on the process of its generation. The work at present focuses on identifying the class (varna) of the phoneme as specified by the Devnagari script. More work is needed to identify the variant of the class identified. Phoneme code thus generated can be used in an application specific way. This work also explains and proves the scientific arrangement of the Devnagari script. This work tries to segment speech into phonemes and identify the phoneme using simple operations like differentiation, zero crossing, FFT. In the phoneme recognition research literature, no work has been reported on Devnagari speech processing. So we consider our work to be the first such attempt in this direction. The process involves extraction of some distinct characteristics of individual phonemes by utilizing Fourier transforms. The system is speaker-independent and is moderately tolerant to background noise.
\end{abstract}

Keywords - Devnagari script, Phonetic speech analysis, Phoneme recognition, Speech to Text conversion.

\section{Introduction}

The work discussed here aims in designing training-less, human independent phoneme class recognition system. This work is not speech recognition or speaker recognition system, but a phoneme recognition system. Phonemes are the basic unit of speech of a language. Each language has its own distinctive set of phonemes, typically numbering between 30 and 50;e.g. English can be represented by a set of around 42 phonemes; Hindi is having a set of 46 phonemes. When different phonemes articulate, voice is produced. Irrespective of a human, the way any phoneme uttered is same and this is the principle of arrangement of the Devnagari script. Though the same speech uttered by different persons is felt different to listen, the information (phonemes in this case) that we extract from the signal is same. This can be because the pattern of vibrations produced by air density on eardrum must be similar for the same phoneme. This work uses the same principle in classifying and identifying the uttered phoneme. The work at present does not deal in identifying the exact phoneme rather its class (Varna).

$$
\mathrm{y}(\mathrm{n})=\mathrm{x}(\mathrm{n})-\mathrm{x}(\mathrm{n}+1)(1)
$$

The work starts after differentiating input speech signal using Eqn. (1). The work is divided in four parts: - 1) End point detection, 2) Segmenting speech into phonemes 3) Phoneme class identification and 4) Phoneme variant identification in the class identified. Out of these, part (1) is not designed to be very robust and accurate, because already the work has been done satisfactorily. Part (2) is implemented using variation in zero-crossing rates and part (3) is implemented using FFT of the speech segment obtained in part (2). Most of the work is focused on parts (2) and (3). No work has been done on implementing Part (4).

\section{The Devnagari Script}

Devnagari script is a script of phonemes arranged in a well structured scientific manner showing unambiguous classification and grouping of phonemes according to the organs used in producing that sound. The letter order of Devnagari is based on phonetic principles which consider both the manner and place of articulation of the consonants and vowels they represent. Accordingly these letters (Akshar) are grouped into different classes called Varnas ("TulyasyaPrayatnam Savarnam") [1]. Every letter and its pronunciation is unique and can't be represented or pronounced by using any other letter(s). This gives us a unique representation for every word uttered by human irrespective of human and context of speech. This feature is absent in languages like English in which one representation and pronunciation of a word or letter can be done in more than one way, e.g. bye, buy both are pronounced similarly. 
The first 25 consonants of Devnagari script, arranged in a 5 X5 matrix, form five different groups of phonemes as in Table 1 Each row of five consonants is generated in totally different way. First four rows are classified depending on the touch point of tongue inside the mouth as Kanthhawya (Velar), Talawya (Palatal), Murdhanya (Retroflex) and Dantawya (Dental). The fifth group is called Aushthawya (Labial) because it is generated using lips only. The elements in a single row are generated using the same organs but varying the time period of touch and pressure at the same or near the touch point of group. Different phonemes in these varnas are Shown in following Table 1.

Table 1 Phonemes of Devnagari script

\begin{tabular}{|l|l|l|l|l|l|}
\hline \multirow{2}{*}{ Phone class } & \multicolumn{5}{|c|}{ Class variant } \\
\cline { 2 - 6 } & \multicolumn{2}{|l|}{ Non-voiced } & \multicolumn{2}{l|}{ voiced } & Nasal \\
\hline Kanthwya & ka & kha & ga & gha & nga \\
\hline Talwaya & cha & chha & ja & jha & nja \\
\hline Murdhanya & ta & tha & da & Dha $^{\wedge}$ & na $^{\wedge}$ \\
\hline Dantawya & ta & tha & da & dha* $^{*}$ & na* $^{*}$ \\
\hline Aushthawya & pa & pha & ba & ma & ma \\
\hline
\end{tabular}

\section{Present work}

The objective of phoneme recognition is to determine zero crossing rates and FFTof the speech signal The steps used in the present phoneme recognition system are discussed below.

\subsection{Input Acquisation}

After capturing the speech by using microphone the speech data is saved in .wav files. For that purpose window XP sound recorder is used. We are using complete list of Devnagari alphabets uttered by 10 different persons ( 5 females +5 males) in normal daily use rooms at $8 \mathrm{kHz}$ with 8 bits per sample. The work being focused mostly on identifying the five classes (varnas) and computing FFT of speech signal to get frequency band of speech signal. The work identifies distinct patterns produced by these five classes and frequency band of speech signal.

\subsection{The Speech Utterance (Data Collection)}

The source of data is a database consisting of 25 characters taken from 5 phone classes and spoken 10 times by 10 speakers; those are 5 males and 5 females of various ages. The data, which is speaker dependent, will be used for further processing.. These characters are recorded by Windows XP sound recorder with sampling rate $8 \mathrm{kHz}, 8$-bit and mono is used to record the utterance.

\section{Speech Processing Work}

The work aims in designing training-less, human independent phonetic speech analysis system to generate phonetic codes of uttered speech. This work is guided by the working of ear in response to audio signals.Speech signals are composed of a sequence of sounds. These sounds and the transitions between them serve as a symbolic representation of information. Though the arrangement of these sounds is governed by the rules of the language, the elemental sounds called phonemes (Akshars) remains the same. Also the way different human produce these phonemes are also same because the difference remains in the parameters of the signal produced like pitch, energy etc. It also is known that the spectral properties of speech waveform such as energy, zero crossings and correlation can be assumed fixed over time intervals on the orders of 10 to $30 \mathrm{~ms}$ [2]. When same speech is uttered by different humans, the information that we extract i.e. phonemes is same irrespective of the speaker. This gave us the question where might this information be present. Hence as first step before starting to work with the speech signal we tried to find some of these parameters that store information of speech. We first converted speech signal into a series of $(+1,0,-1)$ by changing all values above a +ve threshold to +1 , below a - ve threshold to -1 and in between values to 0 . The threshold was selected by manual inspection. The speech was still understandable though was heavy in noise. Next only the points which fell on zerocrossings were marked $+/-1$ according to its sign. The result was same as previous. Next we differentiated the signal using (1) effectively high pass filtering it, as was expected the speech was still preserved. With these results we concluded these two simple parameters viz. zero-crossing and magnitude variation are holding much of the information. Hence these two parameters are always used to further process the signal. We are using complete list of Devnagari alphabets uttered by 10 different persons ( 5 females +5 males) in normal daily use rooms at $8 \mathrm{kHz}$ with 8 bits per sample. The work being focused mostly on identifying the five

classes (varnas), accuracy around $75 \%$ is obtained when speech is composed of consonants from these groups only. The work identifies distinct patterns produced by these five classes. Next we discuss the three parts implemented. 


\subsection{End- Point Detection}

In a same phoneme class it is observed that the first variant is unique in a sense that it is repeated in other three of the four plosive variant except the nasal and is coupled with aspiration and/ or voice bar. Hence even in the absence of this aspiration or voice bar within the marked end points, we can correctly segment and identify the class of the phoneme. Here the technique used to identify endpoints is not very robust and hence low energy nasals and other low energy plosives as specified by [2, pg 132] are tried to be avoided. This limitation is used because already many techniques have already been developed $[4,5]$ and this part is not main focus of the work. Use of one of these techniques is advised. The method used here is inspired by [1,4] and is magnitude based only. The average magnitude envelop of $1^{\text {st }}$ difference of input speech signal is obtained using a rectangular $15 \mathrm{~ms}$ window moved forward at $10 \mathrm{~ms}$ steps. A threshold equal to $15 \%$ of the maximum of this envelop is used to partition this envelop in two parts abvTh and blwTh.In the 1st difference of this magnitude envelope its zero crossings are marked. Zero-crossings nearest to start and end of abvTh is taken as tentative start and end points. As done in [4] we move backward and forward at start and end respectively. The distance of nearest previous/ next ZC to start/ end is computed. If this width is $>3$ start/ end is moved to this point and again we move forward or backward. If we get three consecutive zero-crossings in this 1st difference of amplitude envelop which are not wider than 3 durations of steps and within a threshold of 1 step above or below the width of zero crossing that we started with we finalize the point that we started with as start/ end point. If the difference of widths between current two zero-crossings and the width we started with is $>1$, we move start/ end point to this point and again start to find three consecutive zero-crossings matching above condition. Because the work focuses on identifying the phone class of first 25 phonemes and presence of first phoneme of class in other variants of same class loss of friction (aspiration) before present in weak fricatives $(/ \mathrm{f} /, / \mathrm{th} / / \mathrm{h} /$ ) is not going to affect the result as long as it is segmented correctly that we discuss next

\subsection{Speech Segmentation}

Speech segmentation can be described as a process of identifying boundaries in the speech signal and labeling each of the speech segments between two adjacent boundaries with a symbol. This process of identifying boundaries and labeling can be addressed at various levels of details such as Speech / non-speech segmentation. This is a task of detecting the begin and end of speech in the audio signal. Apart from speech, an audio signal may also contain non-speech data such as music, noise, silence etc. It is important to segment the audio data into speech / non-speech segments, as it acts as pre-processing step for many speech systems such as speech recognition, speaker recognition, etc.,. Output of this process is the boundaries between the speech and non-speech segments in an audio signal

As discussed earlier about experimental observations, it is found that variations in amplitude and the rate of zero- crossings are two of very important parameters that represent the information content of the speech signal. Hence these two parameters are used to segment the speech into its constituent phonemes. The zerocrossings are computed on 1 st difference of the input speech signal with a rectangular window of $15 \mathrm{~ms}$ duration at steps of $10 \mathrm{~ms}$ duration. Similarly the magnitude envelop is computed with same window. After this we marked boundaries with following steps. 1) The positions where the zero-crossing rates in two neighboring cells vary more than $13.30 \%$ are marked. Zerocrossings of nasals and liquids are not found to produce any appreciable change above this threshold so as to get separated from the neighboring phonemes. But magnitude variation is found to show sufficient eparation.Also this segmentation produced multiple segments of longer phonemes like vowels, nasals and fricatives and mixed nasals and liquids with neighboring phonemes. Hence these segments are first mixed using $\mathrm{ZC}$ rate of each segment averaged over $10 \mathrm{~ms}$ and if difference of this $\mathrm{ZC}$ per $10 \mathrm{~ms}$ with neighboring segment is $<=15 \%$ the two segments are mixed.This procedure grouped all the multiple segments of longer phonemes. But these groups still included nasals and liquids. These are separated using amplitude envelop.2) As first step in amplitude based segmentation all the peaks in magnitude envelop that differ from its neighboring valley by $>15 \%$ of maximum of magnitude profile are marked. This is done because when nasals and liquids got mixed with their neighboring phonemes their magnitudes are found to vary appreciably than the neighboring phonemes. Next the parts above and below a threshold of $15 \%$ of maximum of magnitude profile are marked. These segments are then mixed with segments obtained form above step (1).If any of the marked peaks occurs between these segments of speech, this segment is partitioned into above and below the $15 \%$ of average value of this segment. Segments $<=50 \mathrm{~ms}$ or having zero-crossing $>=80$ per $10 \mathrm{~ms}$ is not segmented and is accepted as it is.Once the speech is segmented into phonemes next step is to recognize it.The present work focuses on identifying zero crossing rates of five phoneme classes .The following table shows zero crossing rates of five classes.

\section{Phoneme Recognition}

To identify a phoneme at present we are using a 128 to 512 point FFT depending upon the length of phoneme segment to get the frequency bands of the phoneme. In this part we are computing FFT of the signal 
segment and taking its threshold with a threshold of 0.1. The density is then computed of this threshold of FFT. Here we observed that often for vowels the density is very less compared to non nasal consonants. Nasals as they are closer to vowels [3] are also often found to produce very low density FFT.The 0-4 kHz frequency scale is divided into 17 bands each of $\sim 235 \mathrm{~Hz}$. Each band is numbered from 1 to 17 . Band 1 corresponds to (0 to 235 ) $\mathrm{Hz}$, Band 2 to (235 to 470) Hz and so on. The peak densities of stems in FFT are then computed, normalized and corresponding frequency bands are marked. This density is computed with a rectangular window of size $500 \mathrm{~Hz}$ and in steps of size $200 \mathrm{~Hz}$. These frequency values are first converted into corresponding number of points depending upon FFT size. Because size of FFT is a power of 2 we converted step size to nearest higher power of 2 hence 17 bands widening step size to $\sim 235 \mathrm{~Hz}$. The band with less than $50 \%$ density is discarded assuming a phoneme will produce its bands with strengths $>=50 \%$; this assumption is observed to be a correct decision. The identification of phoneme class is done by finding the positions of peaks thus produced. The phonemes from same group are found to produce these peaks consistently inside a particular band only or a predictable combination of 2 or 3 bands. Retroflex and Dental phoneme classes are found to produce multiple peaks in different bands. Also the positions of FFT peaks of these two groups are found to be very common with each other as can be seen from the TABLE- I.The Retroflex and Dental are hence, in this work, are grouped together. Till now no concrete distinctions have been found between these two groups.Because of these observations artifacts producing more than 3 peaks are ignored to be considered to identify. Also no speech parts are found to be more or less equally distributed on complete frequency range, hence whenever a segment is having more than 9 cells ( $>50 \%$ of total cells) having normalized densities ratio $>=60 \%$ the maximum that segment is marked as noise. Vowels and nasals are found to produce densities $<=5$ most of the times, but this is not a very sharp boundary as vowels also produced densities $>10$ much like non-nasal consonants considerable number of times. Duration of vowels, nasals and fricatives is one of the features to distinguish between these grouped together and non-nasal consonant. Hence if duration of a segment is $>50 \mathrm{~ms}$ that segment is marked as non-nasal consonant and it can be any of vowel, nasal or fricative. Fricatives (SHA) produce very large zero-crossings (> 80 ) hence easy to be identified. All the vowels and phonemes (LA, WA and RA) are occupying band 3 most of the times making them very difficult to be distinguished. FFT peaks for vowels are also producing at most two bands. Fig (1) shows end points detected and the phone boundaries marked by the algorithm. Out of 13 considerable segments 10 are correctly identified. One phoneme "KA" is lost. Overall system accuracy is expected to be $\sim 75 \%$. The positions of largest frequency density groups are as tabulated below.

Table 2 Experimental Observations for Phoneme Class

\begin{tabular}{|l|l|l|}
\hline Class & $\begin{array}{l}\text { Zero } \\
\text { Crossing } \\
\text { Range }\end{array}$ & $\begin{array}{l}\text { Prominent Freq. Band } \\
\text { positions }\end{array}$ \\
\hline Velar & $25-48$ & $(6,7), 5$ \\
\hline Palatal & $45-90$ & 11 to15 \\
\hline Retroflex & $30-60$ & $(3,4)(8,9,10)(12,13,14)$ \\
\hline Dental & $20-55$ & $(3,4)(8,9,10)(12,13,14)$ \\
\hline Labial & $10-55$ & $(2,3,4,5)$ \\
\hline
\end{tabular}
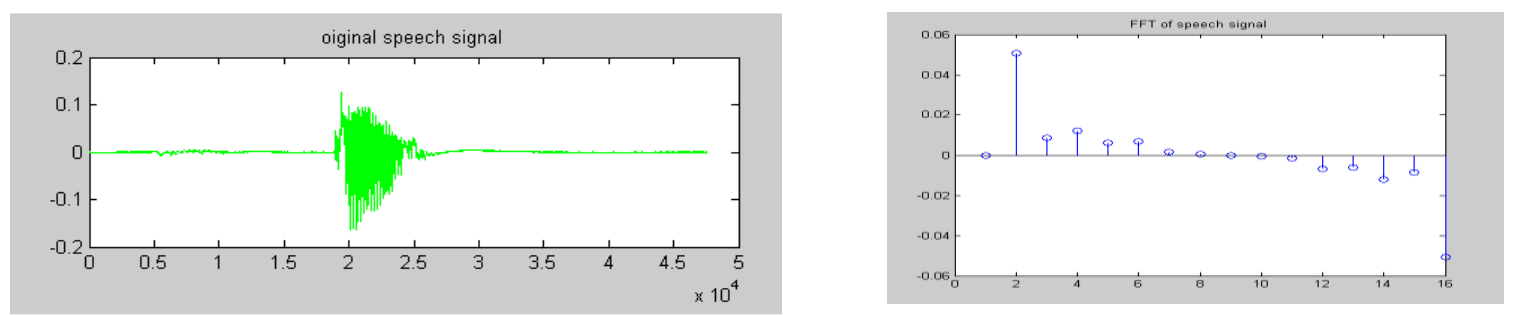

Fig.1 Sample of speech signal of velar class and FFT of Speech signal

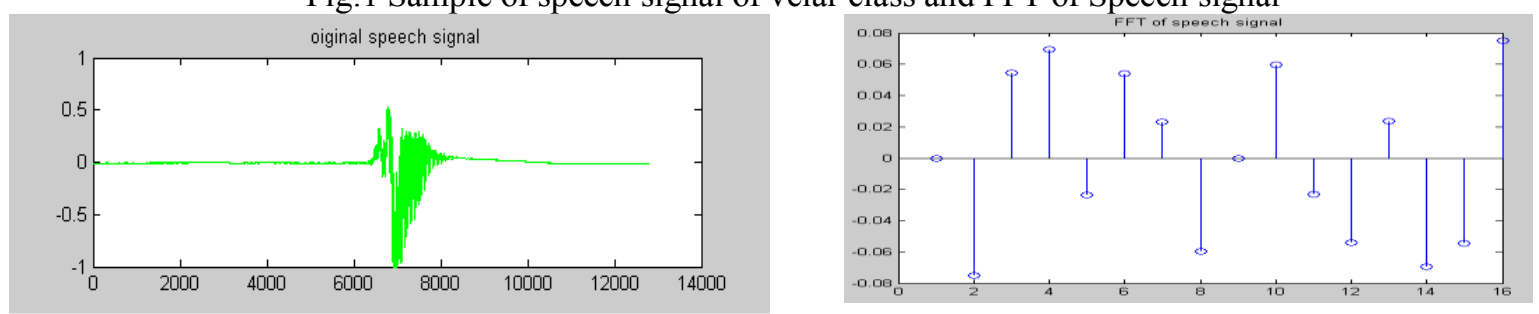

Fig.2 Sample of speech signal of palatal class and FFT of Speech signal 

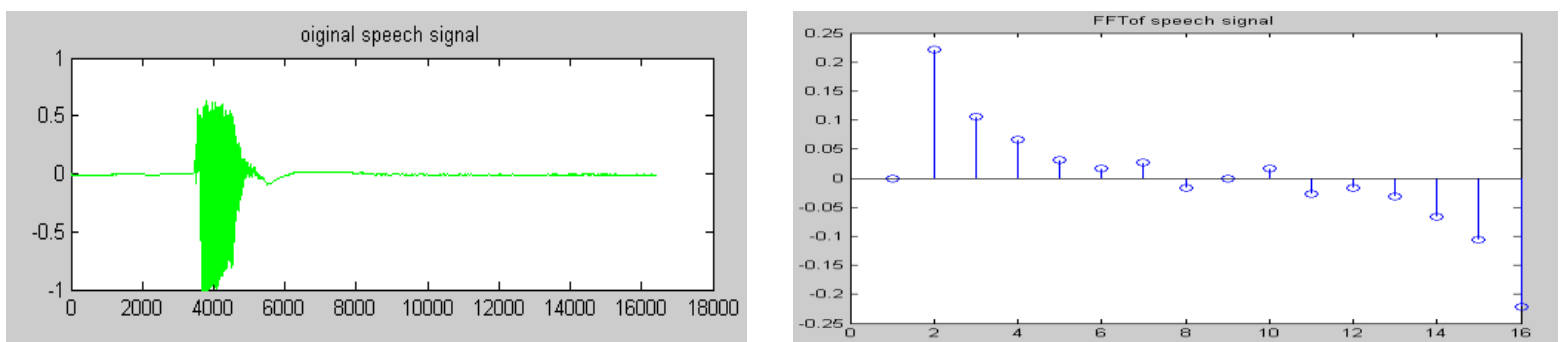

Fig.3 Sample of speech signal of Retroflex class and FFT of Speech signal
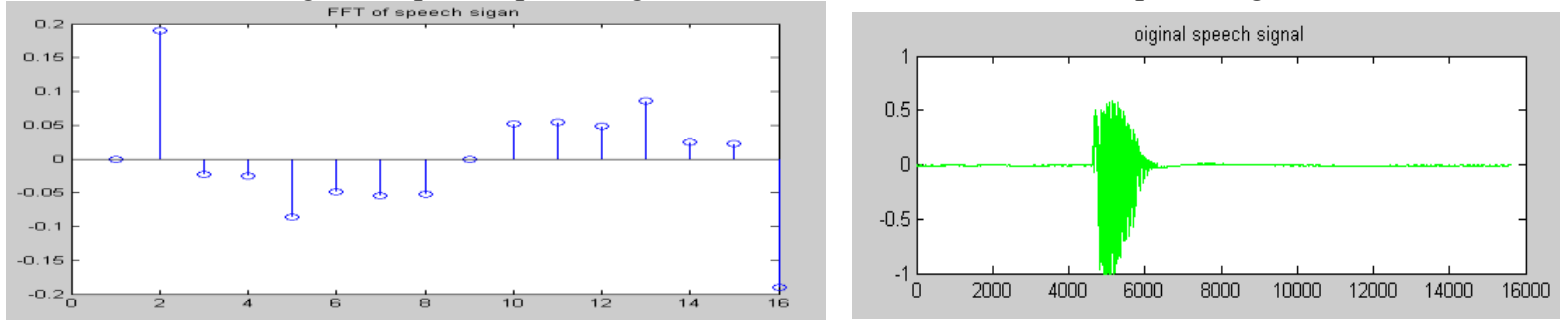

Fig.4 Sample of speech signal of Dental class and FFT of Speech signal
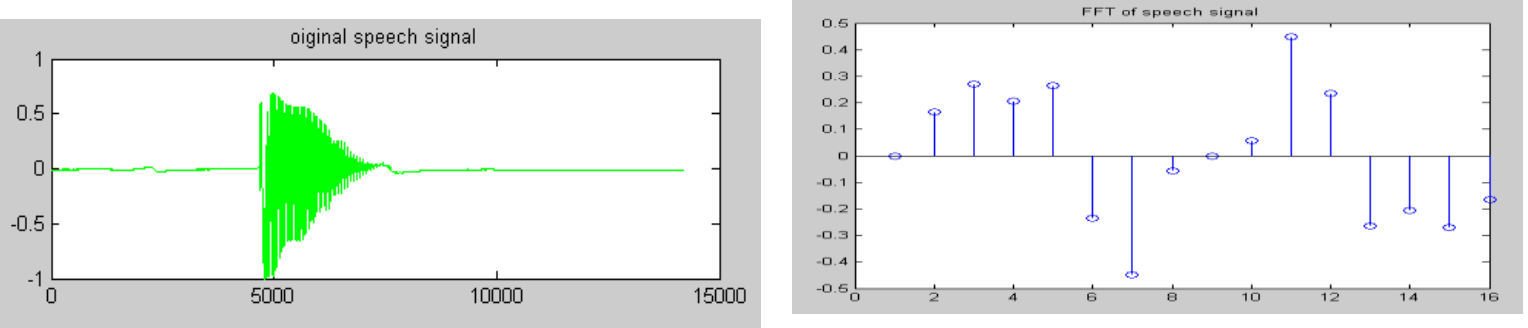

Fig.5 Sample of speech signal of Labial class and FFT of Speech signal

\section{Conclusion}

As was expected in the beginning of the work that a Devnagari script based phoneme recognition system can be designed by considering simple parameters like zero-crossings magnitude. Numbers of samples of speech are taken and generalization is that if zero crossing rates is high, speech signal is unvoiced while if zero crossing rate is low, speech signal is voiced .At present, whatever zero crossing rate, we are getting, its accuracy is not high and some ranges of zero crossings are also overlapping. But we are trying for getting accurate results. FFT of the speech segments is verified.16 point FFT is used to determine to get frequency band of phonemes. At present the accuracy of system is not very high $(\sim 75 \%)$. The work may further be extended to large vocabulary size and to continuous phoneme recognition. The system is sensitive to changing spoken methods and changing scenarios, so the accuracy of the system is a challenging area to work upon. Hence, various Speech enhancements and noise reduction techniques may be applied for making system more efficient, accurate.

\section{Journal Papers:}

\section{References}

[1] Phonetic Speech Analysis for Speech to Text Conversion, 2008 IEEE Region 10 Colloquium and the Third International Conference on Industrial and Information Systems, Kharagpur,INDIA December 8 -10, 2008.

[2] A new approach for phoneme segementation of speech signals, Douglas O'Shaughnessy Ladan Golipour, in Proceedings of Interspeech ,Antwerp,Belgium,August 2007.

\section{Books:}

[1] AshtadhyaeeBhashyam ; Swami Dayanand Saraswati

[2] Digital Processing of Speech Signals; Rabiner, Schafer; Pearson Education.

[3] Discrete Time Speech Signal Processing; Quatieri; Pearson Education.

[4] A Speaker independent digit recognition system, L. Rabiner, M.Sambur.

[5] Robust entropy based endpoint detection, Jia-lin Shen, Jei-Weih Hung,Lin-Shan Lee. 